\title{
Auditorías técnicas de los sistemas de telecontrol implantados en las CCRR realizadas por el Centro Nacional de Tecnología de Regadíos (CENTER)
}

Cervantes Díaz-Toledo, Alfonso ${ }^{1}$, Madurga del Cura, Cristina², Muñoz Sopeña, Daniel ${ }^{2}$, Rodríguez Fernández, Diego $^{2}$, Sánchez de Ribera González, Alejandro², Zazo Salinero, Teresa ${ }^{2}$

1 Ingeniero Técnico de Información de Gestión, TRAGSA - CENTER, acervant@tragsa.es

2 Equipo Técnico del CENTER

\section{Resumen:}

La mejora de la calidad de vida de los agricultores es uno de los objetivos fundamentales de la modernización del regadío y, por ello, se han instalado sistemas de telecontrol que permitan la aplicación de los riegos en el momento adecuado para el cultivo, el control y la gestión de toda la red en su conjunto, es imprescindible para conseguir dicho objetivo.

Estos sistemas de telecontrol funcionan perfectamente en algunas Comunidades de Regantes mientras que, en otras, diferentes motivos han ocasionado que no estén operativos al $100 \%$ o que no se estén aprovechando todas las funcionalidades para las que inicialmente fueron diseñados.

El equipo técnico del CENTER está llevando a cabo auditorías de sistemas completos de telecontrol instalados en distintas Comunidades de Regantes, comprobando in situ su funcionamiento y haciendo propuestas de reparación y mejora para asegurar su pleno rendimiento.

Estas auditorías comprenden los siguientes pasos:

1. Estudio de la documentación de proyecto y ejecución, análisis del sistema, planes de mantenimiento, etc.

2. Realización de pruebas en el laboratorio del CENTER para verificar el correcto funcionamiento de los equipos remotos instalados en la CR.

3. Pruebas en campo y análisis del funcionamiento in situ del sistema y de los diferentes equipos que lo componen.

4. Realización del informe de auditoría en base a los resultados obtenidos en las pruebas de laboratorio y de campo.

El objetivo de este trabajo es, por tanto, mostrar los resultados obtenidos en las auditorías realizadas hasta el momento, destacando la importancia de hacer un seguimiento de estos sistemas ya que, en ocasiones, pequeñas modificaciones o mantenimientos, aseguran un mejor funcionamiento y una mayor eficiencia en la aplicación de los riegos. 